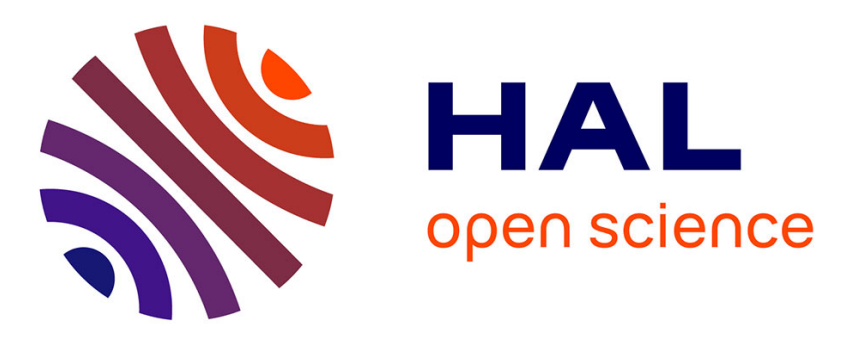

\title{
Superimposition of elastic and non-elastic compression bandages
}

Fanette Chassagne, Clothilde Helouin-Desenne, Jérôme Molimard, Reynald Convert, Pierre Badel, Pascal Giraux

\section{- To cite this version:}

Fanette Chassagne, Clothilde Helouin-Desenne, Jérôme Molimard, Reynald Convert, Pierre Badel, et al. Superimposition of elastic and non-elastic compression bandages. Journal of Vascular Surgery, 2017. hal-01621518

\author{
HAL Id: hal-01621518 \\ https://hal.science/hal-01621518
}

Submitted on 23 Oct 2017

HAL is a multi-disciplinary open access archive for the deposit and dissemination of scientific research documents, whether they are published or not. The documents may come from teaching and research institutions in France or abroad, or from public or private research centers.
L'archive ouverte pluridisciplinaire HAL, est destinée au dépôt et à la diffusion de documents scientifiques de niveau recherche, publiés ou non, émanant des établissements d'enseignement et de recherche français ou étrangers, des laboratoires publics ou privés. 


\section{Superimposition of elastic and non-elastic compression bandages}

2 Fanette Chassagne ${ }^{a, b, c^{*}}$, Clothilde Helouin-Desenne ${ }^{c}$, Jérome Molimard ${ }^{a, b}$,

3 Reynald Convert ${ }^{c}$, Pierre Badel $^{a, b}$, Pascal Giraux ${ }^{d-e}$

4

a Inserm, U1059, Saint-Etienne, F-42023, France

5

${ }^{b}$ Mines Saint-Etienne, Saint-Etienne, F-42023, France

6

${ }^{c}$ Thuasne, BP243, 92307 Levallois-Perret cedex, France

$7{ }^{d}$ Department of Physical Medicine and Rehabilitation, Faculty of Medicine, University 8 Jean Monnet, Saint-Etienne, France

9

${ }^{e}$ Université de Lyon, Université Jean Monnet - Saint-Etienne, LIBM, EA 7424, F-42023, 10 SAINT-ETIENNE, France

11

12

${ }^{*}$ Corresponding

author.

Email:

fanette.chassagne@emse.fr 
14 Objective: To investigate the pressure of superimposed bandages and to compare it to

15 the pressure applied by single component bandages

16 Methods: Six different bandages, composed of one elastic and/or one non-elastic

17 bandages, were applied in a spiral pattern on both legs of 25 patients at risk of venous thrombosis (consecutive to central or peripheral motor deficiency). Pressure was measured at four measurement points on the leg (B1 and C on the medial and lateral sides of the leg) and in three positions: supine, sitting and standing.

Results: The two single bandages applied similar pressure in supine position. Their superimposition showed different pressure levels $(p<0.05)$, but similar static stiffness index, depending on the order in which the bandage components were applied on the leg. The highest interface pressure was measured at point B1 on the medial side of the leg. This point also showed the highest pressure increase from supine to standing position.

27 The pressure applied by the superimposition of two bandages was computed as a

28 linear combination of the pressure applied by each single component (with a constant term set to 0). However, this linear combination did not properly fit the experimental pressure measurements.

Conclusion: The order of bandage application showed a significant impact on interface pressure. However, the poor correlation between the pressure applied by each bandage component and the one resulting from their superimposition underlined the poor understanding of interface pressure generated by the superimposition of compression bandages and should lead to further investigations. 
37 Keywords: Compression bandages, Interface pressure, Multi-layer bandages 
Compression therapy remains the cornerstone of severe venous pathologies such as ulcers [1]. This treatment, whose efficacy is admitted [2]-[4], can be performed thanks to stockings or bandages. Bandages are preferred at the early stages of the treatment

42 [5] and/or for the most severe pathologies. Compression bandages can be differentiated, being either short-stretch or long-stretch [6], with regards to their maximal stretch. Another terminology classifies the bandages with regards to their elastic properties, being either elastic or non-elastic. The difference in mechanical properties will lead to different behaviors once applied on the leg. Elastic bandages result in lower pressure variation from supine to standing position (and also between resting and working pressure) as they can more easily accommodate the change in leg morphology [7]. On the other hand, the pressure increase induced by non-elastic bandages is much higher. From a clinical point of view this differentiation is possible thanks to the Static Stiffness Index (SSI), which is the pressure increase, at measurement point B1 (Figure $1-A$ ), from supine to standing position [8]. This index helps to characterize the behavior of multi-component bandages combining elastic and non-elastic bandages. The superimposition of compression bandages is very common in clinical practice [9] and showed a positive impact on ulcer healing [10], [11]. Multi-layer bandages are often composed of a padding layer (to homogenize the

57 leg geometry), one to two compression layers and possibly a fixation layer (cohesive 58 bandage). Even though the most representative illustration of multi-layer bandages is the so-called 4-layer bandage [12], a large diversity of multi-layer bandages is commercially available [13], [14]. 

generated by one single bandage was extensively investigated. The impact of several parameters such as fabric materials [15]-[17] , application technique [18], [19] or body positions [20] was assessed. However, whether there is a direct relationship between the pressure applied by a single bandage and the one applied by the superimposition of bandages remains an open question.

The pressure applied by two-layer bandages composed of short-stretch and longstretch bandages as well as their stiffness (i.e. the pressure increase per 1 centimeter increase in leg circumference [21]) was investigated in vitro [22]. This pressure applied by superimposed bandages was then compared with the pressure applied by each component separately. Furthermore, in vivo interface pressure measurements were performed to evaluate the stiffness of commercially available multi-component bandages [23]. It was also observed that superimposing bandages led to an increase in

74 Static stiffness Index even with elastic bandages [24]. However, a study performed with the 4-layer bandage, showed that the pressure resulting from the superimposition of bandages was not the sum of the pressure applied by each single bandages [25].

Consequently, the objective of this study was to investigate the pressure applied by the superimposition of elastic and/or non-elastic compression bandages. These pressures were compared to the pressure applied by each single bandage with the aim

81 to evaluate the possible linear correlation between the pressure applied by single and multi-component bandages. The impact of the order of bandage application was also addressed. Interface pressure measurements were performed in 3 positions to assess the pressure variation and the Static Stiffness Index of the bandages. 
86 This protocol was approved by the local Ethical Committee (CPP Sud-Est I - 2015-34)

87 (NCT02803398).

26 patients (16 women -10 men; mean age $=48$ [19 - 72]) were included in the study,

90 but one left after the first visit for medical reasons unrelated to this study. These patients were at risk of venous thrombosis and were treated with compression therapy (stockings or bandages). This risk was the consequence of walking impairment or very limited walking distance induced by a central or peripheral motor deficiency.

They were hospitalized in the Physical Medicine and Rehabilitation Department of the University Hospital of Saint-Etienne, France. To take part in the study, they had to be able to stand for at least $10 \mathrm{~min}$ in a standing frame (Figure $1-\mathrm{B}$ ). Patients with venous thrombosis history, venous or arterial ulcer, cutaneous wound on the lower leg, or with any contraindication to compression therapy were not included in the study. Among the 26 patients included in the study, 13 suffered from post-stroke hemiplegia (partial or complete), 3 suffered from paraplegia (consecutive to a trauma (2) or a surgery (1)) and 2 had a cerebellar stroke. The 8 remaining patients were treated for motor deficiency or impaired balance resulting from various pathologies.

\subsection{Bandages}

104 The pressure applied by two different bandages was investigated in the study: Biflex ${ }^{\circledR}$ 16 (Thuasne) and Rosidal ${ }^{\circledR} \mathrm{K}(\mathrm{L} \& \mathrm{R})$. Both were 10 -cm wide bandages but differed in their mechanical properties. Biflex ${ }^{\circledR} 16$ (B16) is an elastic and long-stretch bandage composed of elastic yarns, whereas Rosidal ${ }^{\circledR} \mathrm{K}(\mathrm{RK})$ is a non-elastic and short-stretch 
bandage only composed of cotton yarns (thus non-elastic yarns). The pressure applied by the different possible combinations of these two bandages was measured, even

110 though these bandages were never associated in regular practice. This resulted in six 111 possible combinations:

- B16: a single Biflex ${ }^{\circledR} 16$ bandage,

- RK: a single Rosidal ${ }^{\circledR} \mathrm{K}$ bandage,

- $\quad$ B16+B16: a Biflex ${ }^{\circledR} 16$ was applied on top of another Biflex ${ }^{\circledR} 16$,

- RK+RK: a Rosidal ${ }^{\circledR} \mathrm{K}$ was applied on top of another Rosidal ${ }^{\circledR} \mathrm{K}$,

- $\quad$ B16+RK: a Rosidal ${ }^{\circledR}$ K was applied on top of a Biflex ${ }^{\circledR} 16$,

- $\quad$ RK+B16: a Biflex ${ }^{\circledR} 16$ was applied on top of a Rosidal ${ }^{\circledR}$ K.

All bandages were applied in a spiral pattern with a $50 \%$ overlapping technique (i.e. a maximum stretch, following their manufacturers' recommendations.

$$
\text { Stretch }=\frac{\text { actual bandage length }(L)}{\text { initial bandage length }\left(L_{0}\right)}
$$

122 Following the methodology described in a previous study [26], the stretch of the applied bandage was then measured thanks to marks drawn every $10-\mathrm{cm}$ on the non-

124 stretched bandage. The six bandages were applied on the leg in a randomized order.

\subsection{Interface pressure measurements}

Interface pressure measurements were performed at four measurement points: two at

127 the height of measurement point B1 (where the Achille's tendon turns into the gastrocnemius muscle [27]) on the medial and lateral side of the leg and two at the 
129 height of measurement point $C$ (at the calf largest circumference [27]) (Figure $1-A$ ).

130 Four probes were kept in place during the six bandage applications. The pressure was

131 measured thanks to the sensor Picopress ${ }^{\circledR}$ (MicroLab Elettronica, Ponte S. Nicolo,

132 Italy), which was used in several previous studies [3], [16], [28].

\section{$133 \quad 2.4$ Interface pressure measurements protocol}

134 Pressure measurements were performed on both legs. The first leg on which bandages

135 were applied was randomly selected for each patient. The order in which the six 136 bandages were applied was also randomized and was the same for both patient's legs.

137 All randomizations were performed with the software Matlab ${ }^{\circledR}$.

138 The protocol was divided into three visits. The time between two visits could not 139 exceed five days. Informed consents were signed by the patients before their 140 inclusion.

$141 \quad \underline{1^{\text {st }} v i s i t}$

142 This visit consisted in the inclusion visit.

$143 \quad \underline{2^{\text {nd }} \text { and } 3^{\text {rd }} \text { visits }}$

144 These two visits, which consisted in interface pressure measurements, were identical:

145 the $2^{\text {nd }}$ visit was performed on the first leg and the $3^{\text {rd }}$ visit on the second leg.

146 First, the patient lied on an examination bed and four sensors were taped on her/his

147 leg. Then the first bandage (selected from the randomization) was applied on the leg.

148 The bandage stretch was measured around measurement points B1 and C after each

149 bandage application and for both bandages in the case of multi-component bandages.

150 Pressure measurement was taken one minute after bandage application. Then the 151 patient sat on the edge of the bed, her/his feet on the ground with a $90^{\circ}$ angle 
152 between the thigh and the lower leg. Pressure was measured one minute later.

153 Eventually, the patient stood in a standing frame (Figure $1-B$ ) and the last pressure

154 measurement was taken after waiting for one min. This waiting time was chosen in

155 order to reach a stationary state of leg venous system [29]. Eventually the patient lied

156 on the examination bed and the same protocol was repeated for the 5 remaining

157 bandages.

\section{$158 \quad 2.5 \quad$ Statistical analysis}

159144 pressure values were measured for each patient, hence a total of 3600 pressure

160 values (105 missing values). Bar graphs represent the mean value and 95\% confidence

161 interval. The normality of the distribution was tested with the Shapiro-Wilk test. Most

162 of the comparison tests were paired tests. For only two groups, the comparison was

163 performed with the non-parametric Wilcoxon test (or the paired T test with regard to

164 the data distribution) and for more than two groups, with the Friedman test. The 165 Nemenyi post-hoc test was used to test the multiple paired comparisons.

166 The coefficient of determination $R^{2}$ was computed as an evaluation of the linear 167 correlation between two samples (the experimental data and the one given by the 168 linear regression for example).

169 The statistical analysis was performed thanks to XLSTAT and Matlab ${ }^{\circledR}$.

\section{$170 \quad 3$ Results}

\subsection{Interface pressure measurements}

172 Stretch of the applied bandage was measured in the area of measurement point B1 173 and C for all bandage applications. Mean stretches, measured at both areas and for all 
174 bandages combinations, were equal to $1.347 \pm 0.005$ for the RK and $1.294 \pm 0.005$

175 for the B16 (whose target stretch was 1.3) (Figure 2). Stretch was higher at 176 measurement point $\mathrm{C}$ than at $\mathrm{B} 1$.

177 Pressures applied by the B16 and the RK at measurement point B1 (medial) in supine 178 position were found to be very similar, respectively $25.69 \pm 1.16$ and $25.94 \pm 1.13$ $179 \mathrm{mmHg}$ (Figure 3). Two-component bandages resulted in much higher pressures: $18049.64 \pm 1.94 \mathrm{mmHg}$ for $2 \mathrm{~B} 16,47.98 \pm 2.24 \mathrm{mmHg}$ for $2 \mathrm{RK}$. The superimposition of 181 two different bandages applied different pressures depending on the order of 182 bandages application ( $p<0.05)$ : $52.38 \pm 2.34 \mathrm{mmHg}$ for a RK applied on top of a B16 183 (B16+RK) and $48.10 \pm 1.59 \mathrm{mmHg}$ for a B16 applied on top of a RK (RK+B16). This 184 difference was statistically significant.

185 Pressure was measured in three positions, supine then sitting and eventually standing, 186 in a very short time (about five minutes). For all bandages, pressure increased from 187 supine to sitting position and then to sitting to standing position (Figure 4 - A).

188 The pressure increase at measurement point B1 (medial) from supine to standing 189 position is the so called Static Stiffness Index (SSI), which helps to characterize the 190 mechanical properties of the whole bandage [8].

191 The minimum SSI was observed for a single elastic bandage (4.18 $\pm 0.75 \mathrm{mmHg}$ ) 192 (Figure 4 - B). However, the superimposition of two of these bandages resulted in an 193 increased SSI $(6.48 \pm 0.82 \mathrm{mmHg})$. The maximum SSI was obtained for the 194 superimposition of two non-elastic bandages $(13.60 \pm 2.29 \mathrm{mmHg})$. As expected, a 195 single non-elastic bandage showed a high SSI (7.35 $\pm 1.55 \mathrm{mmHg})$. Eventually the two 196 combinations of elastic and non-elastic bandages have similar SSI: $9.54 \pm 1.32 \mathrm{mmHg}$ 197 for B16+RK and $9.98 \pm 1.48 \mathrm{mmHg}$ for RK+B16. 
199 Interface pressure was measured at four points on the leg: at the height of 200 measurement point B1 and C on the medial and lateral side of the leg (B1 med, B1 lat, 201 C med and C lat). In supine position, all bandages were found to be degressive (i.e. the 202 pressure applied at measurement point B1 (medial) was higher than at point C 203 (medial)), except the RK (Figure $5-\mathrm{A}$ ). For most bandages, pressures on the lateral 204 side of the leg were lower than on the medial side.

205 The highest interface pressure was always measured at B1 on the medial side of the 206 leg (Figure $5-A, B, C$ ). This measurement point also showed the largest pressure 207 increase from supine to standing position (Figure $5-\mathrm{D}$ ): $8.52 \pm 0.68 \mathrm{mmHg}$ for B1 208 medial, $5.43 \pm 0.65 \mathrm{mmHg}$ for $\mathrm{B} 1$ lateral, $6.42 \pm 0.65 \mathrm{mmHg}$ for $\mathrm{C}$ medial and $2093.63 \pm 0.57 \mathrm{mmHg}$ for C lateral.

\subsection{Pressure applied by a 2-component bandage with regards to the one applied} by each component

212 Interface pressure applied by the six possible combinations of elastic and non-elastic

213 bandages was measured with the aim to better understand the superimposition of compression bandages. The assumption was made that the pressure applied by the

215 superimposition of two bandages would be a linear combination of the pressure 216 applied by both single bandages (with a constant term set to 0 ).

217 The pressure measurements at four locations (height of measurement B1 and C; medial and lateral) on the leg and in supine position were considered for this analysis.

219 First, the ratio between the pressure applied by the superimposition of two identical bandages and the pressure applied by a single bandage was computed. This ratio was 
222 coefficient of determination $\mathrm{R}^{2}$ was very low for the RK.

223 Then the pressure applied by the combination of two different bandages was computed as a linear combination of the pressure applied by both single bandages. By

225 comparing the two equations (Equation $2(c)$ and $(d)$ ), it can be noticed that the order 226 of bandage application tends to impact interface pressure, despite the low coefficient 227 of determination.

$$
\begin{gathered}
P_{2 B 16}=1.89 P_{B 16}\left(R^{2}=0.48, p<0.001\right)(a) \\
P_{2 R K}=1.80 P_{R K}\left(R^{2}=0.06, p<0.001\right)(b) \\
P_{B 16+R K}=1.31 P_{B 16}+0.67 P_{R K}\left(R^{2}=0.37, p<0.001\right)(c) \\
P_{R K+B 16}=0.91 P_{R K}+0.92 P_{B 16}\left(R^{2}=0.10, p<0.001\right)(d)
\end{gathered}
$$

Equation 2: Pressure applied by multi-component bandages as a linear combination of the pressure applied by a single component bandage; $P_{B 16}$ and $P_{R K}$ are the pressures applied by a single B16 and RK, $P_{2 B 16}$ and $P_{2 R K}$ are the pressures applied by the superimposition of two B16 and two RK, $P_{B 16+R K}$ was the pressure applied by a RK over a B16 and $\boldsymbol{P}_{R K+B 16}$ was the pressure applied by a B16 over a RK

\section{Discussion}

229 Interface pressure applied by six different single or multi component bandages was measured at four measurement points on the leg and in three positions. These six bandages, whose SSI were evaluated, resulted from the combination of one elastic

232 (B16) and one inelastic (RK) bandage. The pressure varied with the bandage components but also with the order in which the components were applied on the leg.

234 Eventually, the pressure applied by the four multi-component bandages was computed as a linear combination of the pressure applied by the two single component bandages. 
238 The design of this study was very close to an in vitro study by Hirai et al. [22]. The pressure and the stiffness (i.e. the pressure increase for a $1 \mathrm{~cm}$ leg circumference increase) of different combinations of short and long stretch bandage were measured, as well as the pressure applied by the single bandages. The two single bandages (shortstretch and long-stretch) applied very similar pressure levels (about $30 \mathrm{mmHg}$ ) but had very different stiffnesses: $4 \mathrm{mmHg}$ for the long-stretch bandage and $17 \mathrm{mmHg}$ for the short-stretch bandage.

245 However, their observations contradicted the present study. Indeed, for the range of pressure measured in the present study (about $50 \mathrm{mmHg}$ ), Hirai et al. observed no significant impact of the order of bandage application on in vitro interface pressure and stiffness. In the present study, B16+RK exerted a higher pressure than RK+B16, even though the pressure applied by B16 and RK were similar. Nonetheless the SSI of these two bandages were equal, which was in agreement with the in-vitro study of

251 Hirai et al.. In the present study, the difference between the mean pressures applied by $\mathrm{B} 16+\mathrm{RK}$ and RK+B16 is about $4 \mathrm{mmHg}$. Although it is statistically significant, the clinical meaning of such a difference may be discussed.

This SSI is an usual tool for the classification of compression bandages [6]. The SSI of inelastic bandages is usually higher than $10 \mathrm{mmHg}$ and the one of elastic bandages is

256 lower. However, it was found here that the SSI of RK, which is a non-elastic bandage,

257 was lower than $10 \mathrm{mmHg}$. This was previously observed for a low-pressure bandaging 258 technique [30]. According to this classification, in the present study, all multi259 component bandages composed of at least one non-elastic bandage are inelastic 260 bandages. This result corroborated the fact that adding at least one non-elastic 

superimposition of bandages (either elastic or non-elastic) increased the SSI, which led

263 to think that bandage-to-bandage friction can play a role in the SSI. Indeed, by superimposing bandages, the bandage-to-bandage contact surface is highly increased. In standing position, the increase in leg volume is limited by the mechanical resistance of the bandage but also by the friction between the different layers.

Bandage degressivity was assessed thanks to interface pressure at 4 different measurement points (points B1 and $\mathrm{C}$ on the medial and lateral side of the leg). Compression bandages are commonly applied on the leg with a constant stretch. The conical shape of the leg (increase circumference from the ankle to the knee) lead to a

271 degressive pressure profile: the pressure decreases from the ankle to the knee. In this

272 study, all bandages were found to be significantly degressive except the RK. This can

273 be explained by the fact that stretch was higher at measurement point $C$ than at point

274 B1 (Figure 2). As a consequence, as it can be noticed in Figure 6, this stretch increase

275 (in green in Figure 6) led to a larger tension increase for the RK than for the B16, 276 respectively $48.3 \%$ and $9.0 \%$ of the tension for the mean stretch (in black in Figure 6).

277 The difference in stretch between the two heights on the leg (heights of measurement

278 point B1 and C) led to a much higher increase in tension for the RK than for the B16.

279 For RK, this larger tension increase may compensate the increase in circumference 280 from measurement point B1 to $C$, hence the fact that the bandage was not degressive. 281 Nonetheless, all the trends observed here about the bandage stretch cannot be 282 generalized as they are the results of only one bandager.

283 Eventually, measuring the pressure at two heights on the leg and on the medial and lateral sides of the leg showed that the maximum pressure increase from supine to 
standing position was observed at measurement point B1 which confirmed the

relevance of the use of this point for the characterization of the stiffness of the

287 bandage [30]. Also, pressures measured on the medial side of the leg were higher than those measured on the lateral side. This can easily be explained by the anatomy of the

289 leg: the radii of curvature are lower on the medial side than on the lateral side.

The pressure applied by two-component bandages at four measurement points on the

293 leg (in supine position) was computed as a linear combination of the one applied by each single component. However, except for the superimposition of two elastic compression bandages, this linear model did not properly fit the experimental data.

It could have been expected that the pressure applied by a two-component bandage would be the sum of the pressures applied by each single component bandage 298 (according to Laplace's Law). A possible explanation could be the thickness of the bandage [31]. Moreover, the second bandage was applied on a deformed leg shape

300 induced by the application of the first bandage.

301 For the computation of the pressure applied by two-component bandages, setting the 302 constant term to 0 for the linear model might be a too strong hypothesis. Also, the low correlation between the pressure applied by a two-component bandage, composed of

304 at least one non-elastic bandage, and the one applied by a single component bandage might be due to the mechanical properties of the fabrics. As it can be observed in 306 Figure 6, the stretch variation in between the confidence interval (in red in Figure 6) 
307 induced a much larger tension variation for the non-elastic bandage (about $19 \%$ of the

308 mean value) than for the elastic bandage (about $4 \%$ of the mean value).

309 Finally, the very low correlation between the pressure applied by each single 310 components and the one resulting from their superimposition highlighted the lack of

311 understanding of the mechanisms involved in bandages superimposition.

\subsection{Limitations}

313 Pressure measurements were taken in a very short time after bandage application.

314 However pressure tends to vary over time [32] because of various phenomena: such as

315 bandage relaxation [33] (loss of tension over time), reduction of leg edema [34] and

316 bandage slippage on the lower leg. It was chosen to take measurements in a very short

317 time to limit the impact of these phenomena, which are complex to evaluate.

318 Nevertheless, relaxation tests (performed in our laboratory, results not shown here)

319 showed that after 10 minutes, the B16 lost about 7\% of its nominal tension (for a

320 stretch equal to 1.30 ) whereas the RK lost about $22 \%$ (for a stretch equal to 1.35 ). It

321 could be interesting to perform these measurements within a longer period of time to

322 reach a stationary state for bandage materials, although it would hardly be sustainable

323 for the patients. Moreover, these measurements were static measurements. Even

324 though pressure was measured in three positions, this study did not investigate the

325 working pressure of these bandages (i.e. the interface pressure applied while walking).

326 The two bandages were chosen as representative bandages of elastic and non-elastic

327 bandages, even though they are not routinely combined in usual clinical practice.

328 Thereby measurements of pressure applied by other commercially available multicomponent bandages would be of high interest. 
330 Patients included in the study were all at risk of venous thrombosis due to walking

331 impairment. However, the causes of their motor deficiency were very heterogeneous.

332 Some of the pathologies might have had an impact of patients' muscle pump, which

333 could influence pressure variations in different body positions. In future studies, it

334 would be relevant to assess the venous pumping function of patients' leg before 335 bandage applications.

\section{Conclusion}

337 This study consisted in static interface pressure measurements applied by 6 different

338 bandages, all composed of elastic and/or non-elastic bandages. First, it was observed

339 that the components of the bandage but also the order in which they are applied on

340 the leg significantly impact interface pressure. Second, the very low correlation

341 between the pressure applied by multi-component bandages and the one applied by

342 the single-component highlighted the poor understanding of the mechanisms involved

343 in bandages superimposition. Further mechanical studies would be needed to better

344 understand the pressure generation resulting from such superimposition.

345 Following a similar protocol, it would be clinically relevant to characterize the 346 performance of commercially available multi-component bandages, and also to 347 investigate their dynamic behavior, while walking for instance.

\section{$348 \quad 6$ Conflict of Interest}

349 Thuasne is a compression bandages manufacturer. 
352 [1] European Wound Management Association, "Position document: Understanding compression therapy." London: MEP Ltd., 2003.

[2] F. Amsler, T. Willenberg, and W. Blättler, "In search of optimal compression therapy for venous leg ulcers: A meta-analysis of studies comparing divers bandages with specifically designed stockings," J. Vasc. Surg., vol. 50, no. 3, pp. 668-674, Sep. 2009.

[3] C. R. Lattimer, E. Kalodiki, M. Kafeza, M. Azzam, and G. Geroulakos, “Quantifying the Degree Graduated Elastic Compression Stockings Enhance Venous Emptying," Eur. J. Vasc. Endovasc. Surg., vol. 47, no. 1, pp. 75-80, Jan. 2014.

[4] O. Agu, G. Hamilton, and D. Baker, "Graduated compression stockings in the prevention of venous thromboembolism," Br. J. Surg., vol. 86, no. 8, pp. 9921004, Aug. 1999.

[5] F. Allaert, "Différentes indications de la compression élastique," Actual. Pharm., vol. 54, no. 547, pp. 14-20, Jun. 2015.

[6] H. Partsch et al., "Classification of Compression Bandages: Practical Aspects," Dermatol. Surg., vol. 34, no. 5, pp. 600-609, May 2008.

[7] J. Dissemond et al., "Compression therapy in patients with venous leg ulcers: Compression in leg ulcers," J. Dtsch. Dermatol. Ges., vol. 14, no. 11, pp. 10721087, Nov. 2016.

[8] H. Partsch, "The static stiffness index: a simple method to assess the elastic property of compression material in vivo," Dermatol. Surg., vol. 31, no. 6, pp. 625-630, Jun. 2005.

[9] J. Hafner, I. Botonakis, and G. Burg, "A comparison of multilayer bandage systems during rest, exercise, and over 2 days of wear time," Arch. Dermatol., vol. 136, no. 7, pp. 857-863, Jul. 2000.

[10] S. O'Meara, N. Cullum, E. A. Nelson, and J. C. Dumville, "Compression for venous leg ulcers," in Cochrane Database of Systematic Reviews, The Cochrane Collaboration, Ed. Chichester, UK: John Wiley \& Sons, Ltd, 2012.

[11] D. J. Milic et al., "The influence of different sub-bandage pressure values on venous leg ulcers healing when treated with compression therapy," J. Vasc. Surg., vol. 51, no. 3, pp. 655-661, Mar. 2010.

[12] C. Moffatt, "Four-layer bandaging: from concept to practice," Int. J. Low. Extrem. Wounds, vol. 1, no. 1, pp. 13-26, Mar. 2002.

[13] R. Hanna, S. Bohbot, and N. Connolly, "A comparison of interface pressures of three compression bandage systems," Br. J. Nurs. Mark Allen Publ., vol. 17, no. 20, pp. S16-24, Nov. 2008.

[14] I. K. Y. Wong et al., "Randomized controlled trial comparing treatment outcome of two compression bandaging systems and standard care without compression in patients with venous leg ulcers," J. Eur. Acad. Dermatol. Venereol., vol. 26, no. 1, pp. 102-110, Jan. 2012.

[15] L. Danielsen, S. Munk Madsen, L. Henriksen, J. Sindrup, and L. J. Petersen, "Subbandage pressure measurements comparing a long-stretch with a shortstretch compression bandage," Acta Derm Venerol, vol. 78, pp. 201-204, 1998. 
[16] D. Rimaud, R. Convert, and P. Calmels, "In vivo measurement of compression bandage interface pressures: The first study," Ann. Phys. Rehabil. Med., vol. 57, no. 6-7, pp. 394-408, Aug. 2014.

[17] M. Hirai, "Changes in interface pressure under elastic and short stretch bandages during posture changes and exercises," Phlebol. - Venous Forum R. Soc. Med., vol. 13, pp. 25-28, 1998.

[18] A. Coull, D. Tolson, and J. Mclntosh, "Class-3c compression bandaging for venous ulcers: comparison of spiral and figure-of-eight techniques," J. Adv. Nurs., vol. 54, no. 3, pp. 274-283, May 2006.

[19] J. P. Benigni, J. F. Uhl, A. Cornu-Thénard, and E. Blin, "Compression bandages: influence of techniques of use on their clinical efficiency and tolerance," Int. Angiol., vol. 27, no. 1, pp. 68-73, Feb. 2008.

[20] A. J. Lee, J. J. Dale, C. V. Ruckley, B. Gibson, R. J. Prescott, and D. Brown, "Compression therapy : effects of posture and application techniques on initial pressures delivered by bandages of different physical properties," Eur. J. Vasc. Endovasc. Surg. Off. J. Eur. Soc. Vasc. Surg., vol. 31, no. 5, pp. 542-552, May 2006.

[21] CEN European Prestandard, "Medical compression hosiery." Aug-2001.

[22] M. Hirai, A. Koyama, K. Miyazaki, H. Iwata, and Y. Kominami, "Interface pressure and stiffness in different combinations of compression material," Phlebology, vol. 27, no. 2, pp. 82-89, Sep. 2011.

[23] G. Mosti and V. Mattaliano, "Simultaneous changes of leg circumference and interface pressure under different compression bandages," Eur. J. Vasc. Endovasc. Surg., vol. 33, no. 4, pp. 476-482, Apr. 2007.

[24] G. Mosti, V. Mattaliano, and H. Partsch, "Influence of Different Materials in Multicomponent Bandages on Pressure and Stiffness of the Final Bandage," Dermatol. Surg., vol. 34, no. 5, pp. 631-639, May 2008.

[25] J. J. Dale, C. V. Ruckley, B. Gibson, D. Brown, A. J. Lee, and R. J. Prescott, "Multilayer Compression: Comparison of Four Different Four-layer Bandage Systems Applied to the Leg," Eur. J. Vasc. Endovasc. Surg., vol. 27, no. 1, pp. 94-99, Jan. 2004.

[26] F. Chassagne, F. Martin, P. Badel, R. Convert, P. Giraux, and J. Molimard, "Experimental Investigation of Pressure Applied on the Lower Leg by Elastic Compression Bandage," Ann. Biomed. Eng., vol. 43, no. 12, pp. 2967-2977, Dec. 2015.

[27] H. Partsch et al., "Measurement of lower leg compression in vivo: recommendations for the performance of measurements of interface pressure and stiffness: consensus statement," Dermatol. Surg., vol. 32, no. 2, p. 224-232; discussion 233, Feb. 2006.

[28] R. J. Damstra and H. Partsch, "Prospective, randomized, controlled trial comparing the effectiveness of adjustable compression Velcro wraps versus inelastic multicomponent compression bandages in the initial treatment of leg lymphedema," J. Vasc. Surg. Venous Lymphat. Disord., vol. 1, no. 1, pp. 13-19, Jan. 2013.

[29] M. H. Meissner et al., "The hemodynamics and diagnosis of venous disease," J. Vasc. Surg., vol. 46, no. 6, pp. S4-S24, Dec. 2007. 
[30] H. Partsch, "The use of pressure change on standing as a surrogate measure of the stiffness of a compression bandage," Eur. J. Vasc. Endovasc. Surg., vol. 30, no. 4, pp. 415-421, Oct. 2005.

[31] J. Al Khaburi, E. A. Nelson, J. Hutchinson, and A. A. Dehghani-Sanij, "Impact of multilayered compression bandages on sub-bandage interface pressure: a model," Phlebology, vol. 26, no. 2, pp. 75-83, Mar. 2011.

[32] I. K. Y. Wong, M. B. L. Man, O. S. H. Chan, F. C. Siu, M. Abel, and A. Andriessen, "Comparison of the interface pressure and stiffness of four types of compression systems," J. Wound Care, vol. 21, no. 4, pp. 161-167, Apr. 2012.

[33] B. Kumar, A. Das, and R. Alagirusamy, "Effect of material and structure of compression bandage on interface pressure variation over time," Phlebol. Venous Forum R. Soc. Med., Apr. 2013.

[34] R. J. Damstra, E. R. Brouwer, and H. Partsch, "Controlled, comparative study of relation between volume changes and interface pressure under short-stretch bandages in leg lymphedema patients," Dermatol. Surg., vol. 34, no. 6, pp. 773778-779, Jun. 2008. 


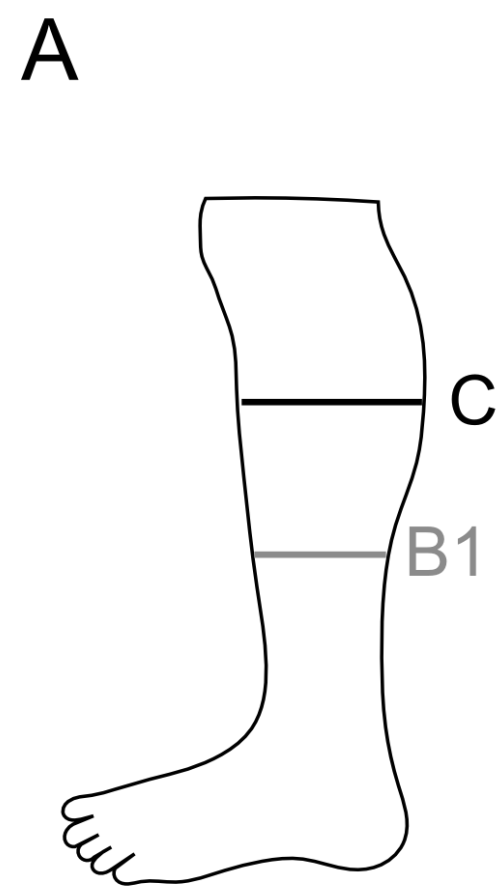

457
B

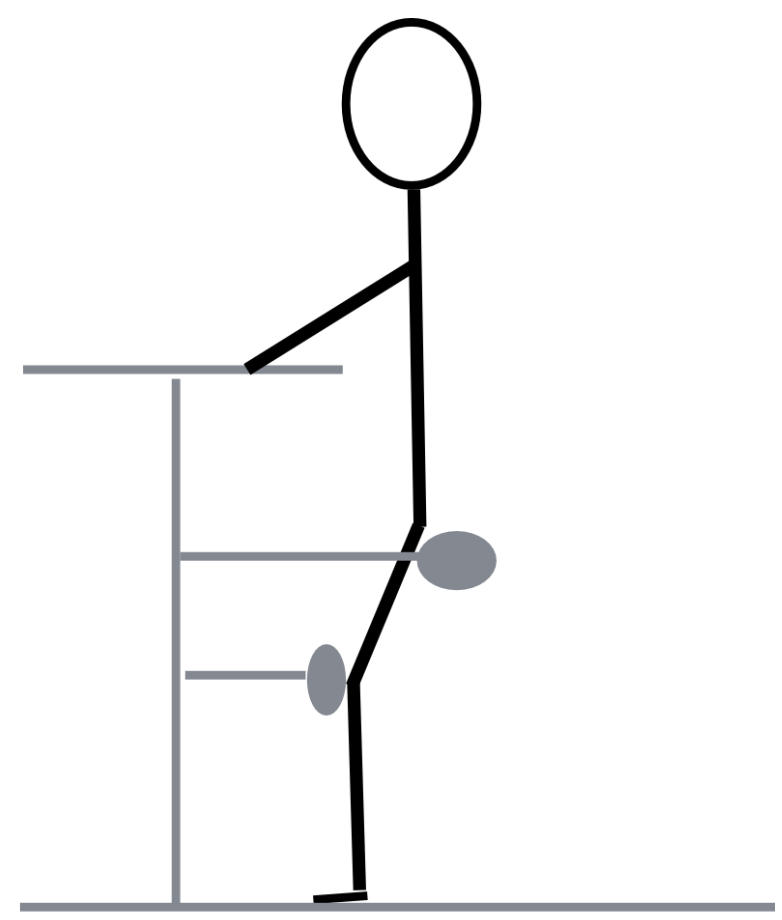

Standing frame 


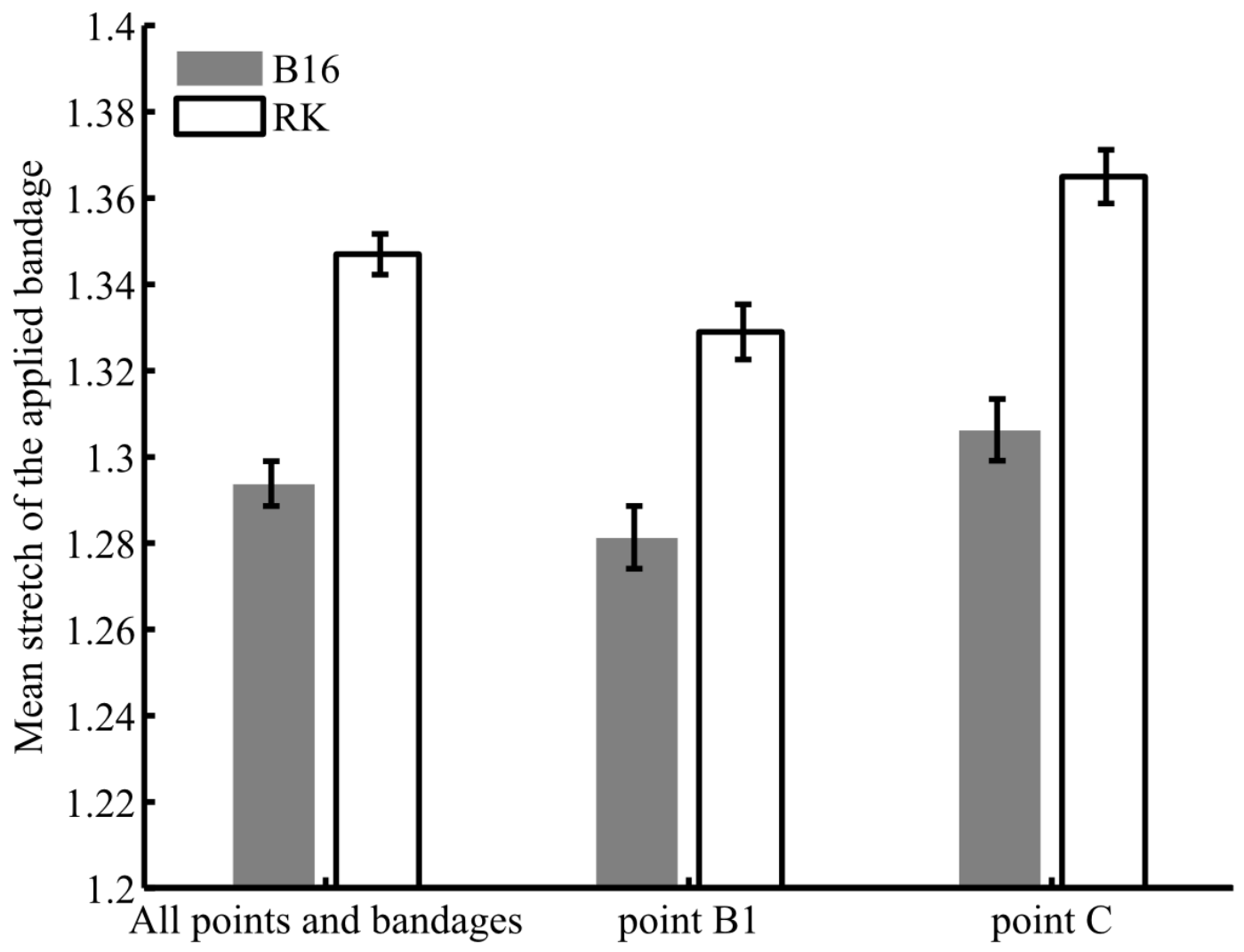

459

Figure 2: Stretch of the applied bandages; for all measurement points and bandages; at measurement point B1; at measurement point $C_{\text {; }}$ 


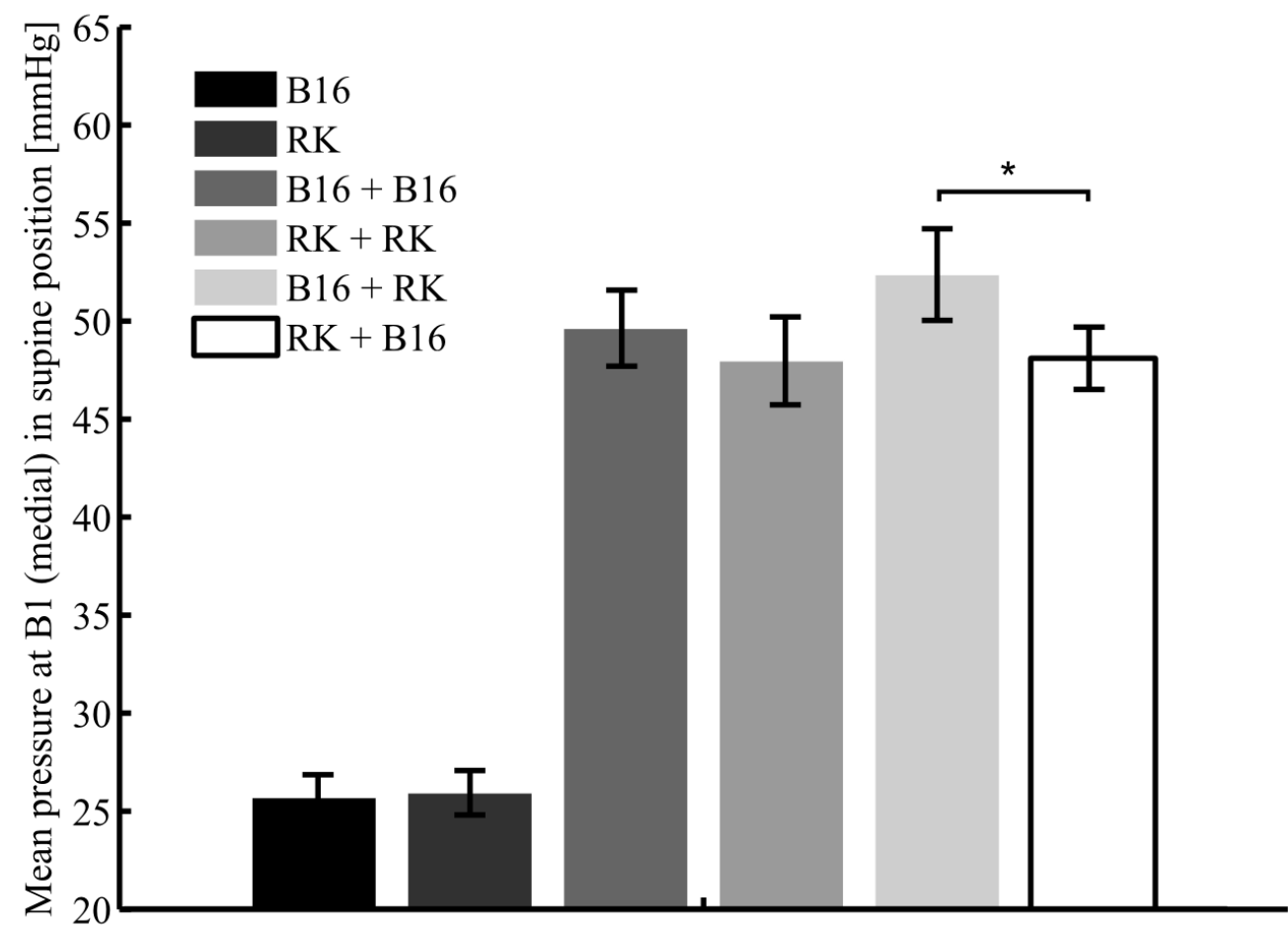


A

467

468

469
B

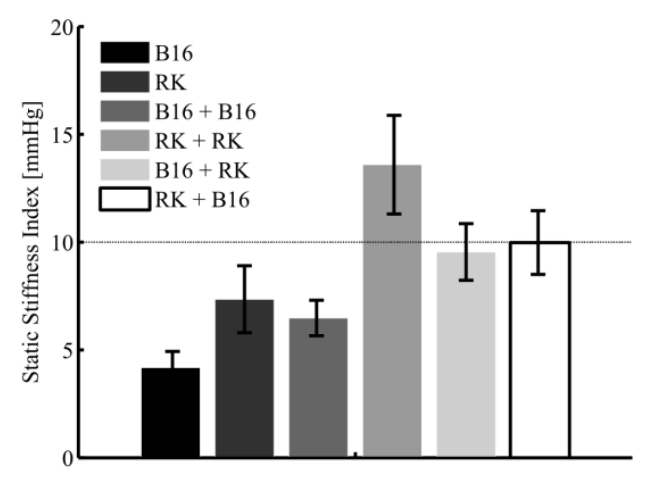

Figure 4: A - Mean pressures applied by the 6 bandages at measurement point B1 (medial) in supine, sitting and standing positions; n.s. states for non-significant difference; B - Static Stiffness Index for the 6 bandages 

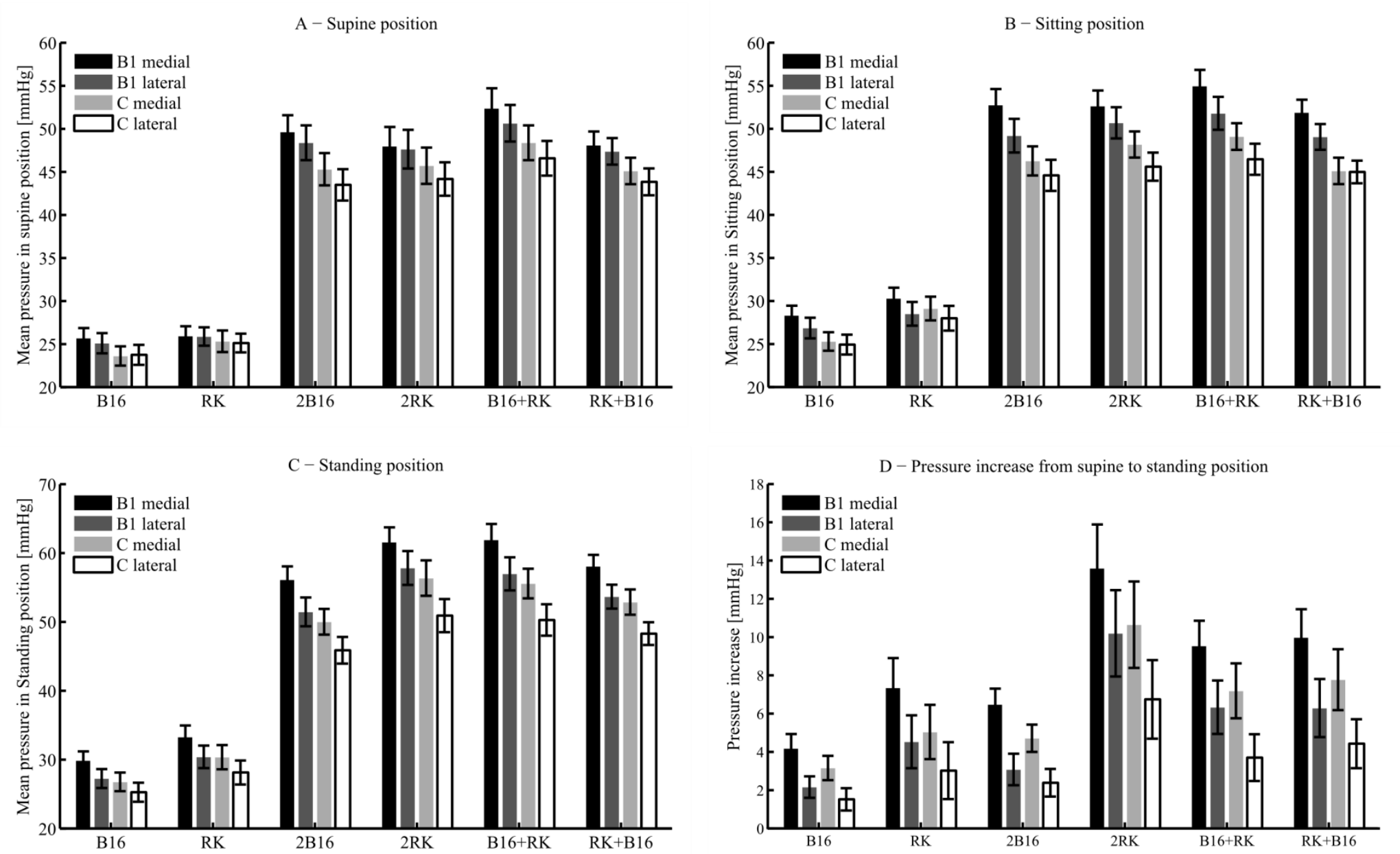

Figure 5: Mean pressure for the four measurement points and the six bandages in the three positions, supine (A), sitting (B) and standing (C); Pressure increase from supine to standing 

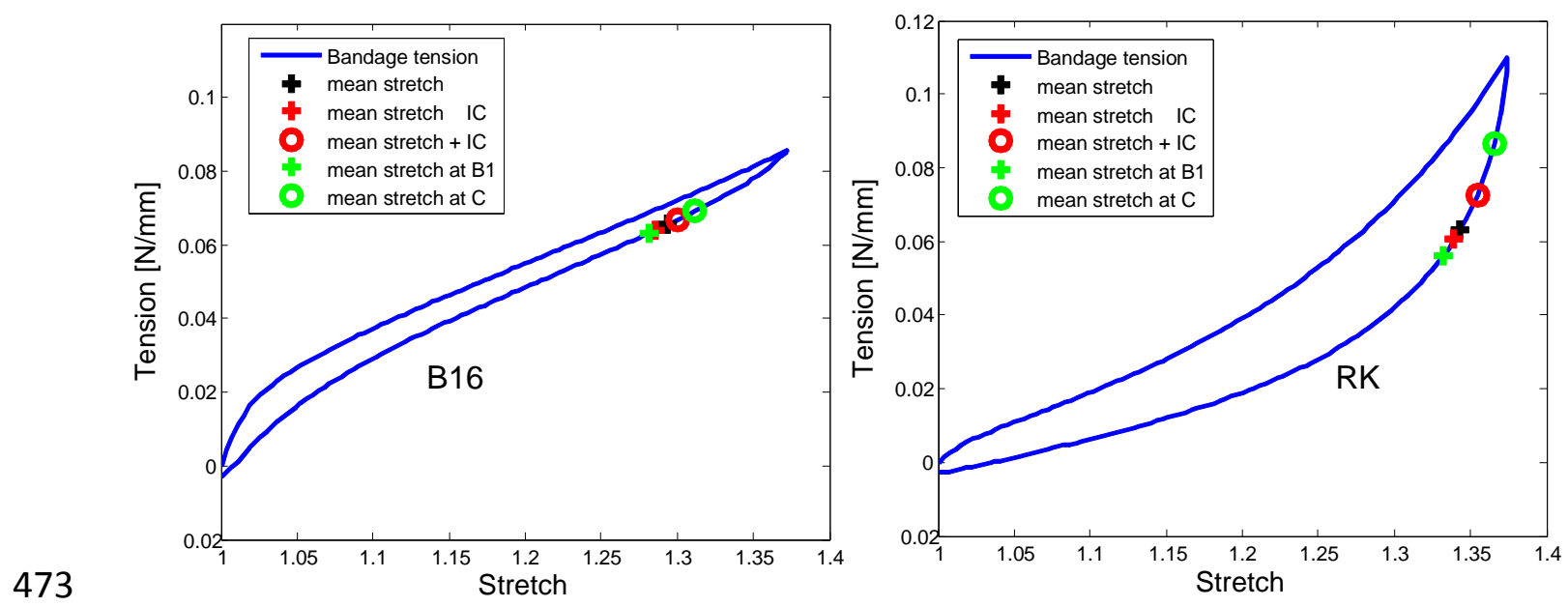

474 Figure 6: Tension as a function of the stretch for both bandages B16 and RK

475 